\title{
Analysis of High-Speed Railway Communication Technology and Prospect of LTE-R Technology
}

\author{
Zihao $\mathrm{Fu}^{1, \mathrm{a}}$
}

\author{
${ }^{1}$ School of North China Electric Power University, Beijing 102206, China; \\ a18810555907@163.com
}

\begin{abstract}
In recent years, the rapid development of high-speed rail technology in China has not only promoted the development of the economy, but also made our country's transportation more developed. High speed rail trains up to $350 \mathrm{~km} / \mathrm{h}$ per hour, so communication technology has been a technical difficulty in the field of high-speed rail. High demand for communication technology. This paper discusses the shortcomings of the existing digital mobile communication system in our country, and analyzes the characteristics of the LTE-R system and looks forward to its future.
\end{abstract}

Keywords: LTE-R technology; Related research; Effective application.

\section{高铁通信技术分析与 LTE-R 技术展望}

\author{
富子豪 $^{1}$
}

(1. 华北电力大学 电气与电子工程学院, 北京市 102206)

摘要: 近年来, 高铁技术在我国飞速发展, 不仅促进了各地经济的发展, 而且使得我国交通进一步发达。高铁列车的最 高时速可达 $350 \mathrm{~km} / \mathrm{h}$, 因此通信技术在高铁领域一直是一个技术难点。对通信技术有较高的要求。本文论述了我国现有的数 字移动通信系统的不足, 并对 LTE-R 系统的特点进行分析以及展望其未来。

关键词: LTE-R 技术; 相关研究; 有效应用

中图分类号：TN929.5 文献标志码：A

\section{引言}

通信技术在 1960 年左右就开始广泛应用与我国的铁路交通领域中，至今已经有五十多年发展历史。 随着高速铁路在我国的迅速发展, 尤其是高铁技术近年来的发展, 列车最高时速可达 $350 \mathrm{~km} / \mathrm{h}$, 高速运行 的列车条件下, 原有通信系统已经无法满足现有的要求。

GSM-R（铁路专用数字移动通信系统）技术是欧洲专为铁路开发的移动通信系统, 它能够应用于高速 和一般类车上，具有价格低廉，性能可靠的优点。目前其已占据国内外铁路的无线通信领域的主导地位。 该通信系统属于窄带通信系统, 带宽窄, 频谱利用较低, 承载的数据速率较低, 很难满足现代高铁列车的 通信。

国际铁路联盟提出, 目前铁路系统应跨越现有技术, 向 $4 \mathrm{G}$ 时代迈进。4G 即是 LTE 技术的简称, 并计 划在近期制定下一代铁路通信系统标准。中国是最先提出发展 LTE-R（铁路专用数字移动通信系统）技术 的国家。LTE-R 技术相较 GSM-R 而言, 具有诸多优势, 如具有更高的安全性和可靠性, 且成本更低。

目前我国设立了 “基于 TD-LTE 的高速铁路宽带通信的关键技术研究与应用验证” 的研究课题, 我国 铁路通信系统从 GSM-R 向 LTE-R 的演进已经成为主流思路。近年来 GSM-R 技术发展迅速, 但已经接近饱和, LTE 技术必将取代现代的主流 GSM-R 技术。 
本文将首先分析现阶段高铁通信的基本需求，并对 LTE-R 系统的关键技术进行理论上的分析，并对该 技术用于未来我国高铁通信系统做出展望。

\section{1 铁路通信系统现状与局限性}

\section{1 现有系统的应用模式}

我国铁路交通领域中，通信技术在上世纪六十年代就已经投入应用了。随着高铁技术的飞速发展，高 铁中的通信技术已经不再局限于传统的移动联系、基础应急通信、区间调度等作用。高铁要求通信技术可 以高效地监控和传输各种数据, 但其核心仍是人对高速行驶的列车进行管理。我国通信技术在铁路领域的 应用经历了三个阶段，分别是模拟通信阶段、集群通信阶段及到现在的 GSM-R 网络通信阶段。

现阶段，我国的 GSM-R 系统已经建成，其是一种基于目前世界最通用成熟的基于 GSM 平台上的，为满 足铁路应用开发的数字模式的无线通信系统。GSM-R 的发展也经历了三个阶段, 分别是技术试验阶段, GSM-R 系统测试阶段，及最后的工程实现阶段。在采用该系统的进程中，经历了如下三个阶段：第一阶段，全面 建设高铁信息化，通信系统与移动系统能够实现同步; 第二阶段 GSM-R 网络与高铁 CTC 系统同步; 第三阶 段我国新建的高铁项目要先与 GSM-R 完成同步，不能重复建设。

我国是采用 GSM-R+FAS（固定用户接入系统）相互连通的方式，来实现有线与无限调度通信。调度台 和值班台等有线终端属于 FAS 一端, $\mathrm{OPH}$ (移动手持台) 和 CIR（机车台）属于移动一端。在 MSC（移动交 换中心）与 FAS 间，采用相应的协议接口，从而实现无线系统与有线系统间的互通。具体结构如图 1 所示。

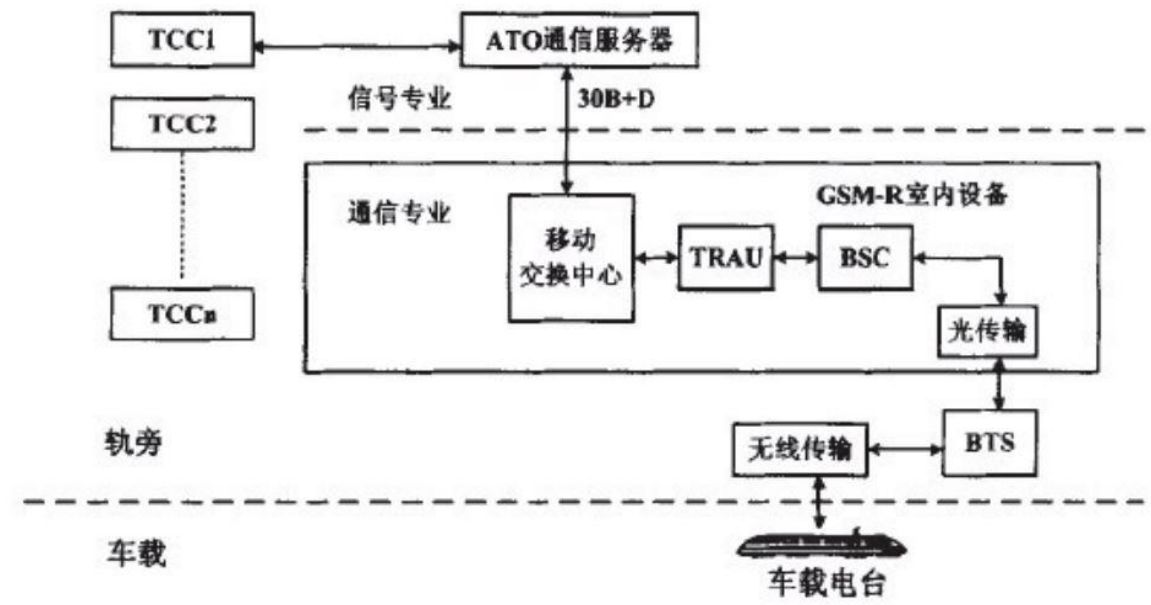

图 1 GSM-R+FAS 方式组网

由该图可知, 调度台、车站台以及其他固定的终端接入 FAS 端, CIR 和 OPH 通过空口接入 MSC, FAS 与 MSC 之间通过 DSSI 互相联通。

\section{2 现有 GSM-R 系统的局限性}

现在我国使用的较成熟的 GSM-R 技术及其组成的 GSM-R+FAS 系统，都有着较大的局限性。尤其是我国 高铁建设的步伐正不断加快, 对控制调度、安全监控、乘客通信等诸多方面都有着更高的要求。现有的 GSM-R 技术在发展中遇到了几方面的问题:

1） GSM-R 通信频谱较窄, 上下行频谱平均值只有 4Mhz 左右。这就限制了 GSM-R 的通信速率较低, 很 难满足高铁对于数据传输的需求。

2) 因通信制式对于 GSM-R 系统的影响, GSM-R 系统有较高的延迟。 
3） GSM-R 系统容易受到环境和高铁列车速度变化的影响, 其可靠性和稳定性并不是很好, 高铁运行的 速度快, 线路较长, 运行环境较为复杂, 会面临很多复杂的地形因素, GSM-R 在遇到地形障碍时的传输效 果较差。

现有 GSM-R 技术与 FAS 共同构建的系统也存在一定的局限性，主要体现在以下几个方面:

1) FAS 系统与无线调度通信系统相对独立, 从系统建设、运行管理与系统维护上来看, 两个系统相对 独立，会给系统的各个环节的管理等带来一定的复杂性。

2) 无线、优先系统相对独立, 各有业务控制、信息交换核心。对于各种新业务的规划和实施, 需要 协调各有线无线系统的环节，规划到实施再到成熟的应用周期较长。

3) FAS 系统部分采用电路域的交换技术。该技术在传统语音通信领域有固有的优势, 但是在其他应用, 如视频应用中有较大的限制。

\section{LTE-R 系统}

\section{1LTE-R 特征与关键技术}

相比于 GSM-R 系统, LTE-R 通信技术具有数据通信速率较高、业务实时性好、载波带宽灵活等特点, 符合高铁现阶段的业务需求，推进铁路行业进入到一个新的发展阶段。

LTE-R(Long Term Evolution for Railway) 技术主要由系统指标、网络质量指标以及业务质量指标三 个部分组成, 系统指标包含宽带、频段、峰值速率以及移动性; 网络指标包含覆盖、容量、保持特性指标、 接入特性指标、移动特性指标及时延; 业务质量指标包含业务优先级、业务时延与数据包丢失率等。

LTE-R 技术的主要特征如下:

1） LTE-R 能够配置 $1.25 \mathrm{MHz}$ 到 $20 \mathrm{MHz}$ 带宽, 因此 LTE 网络可以提供 $100 \mathrm{M}$ 以上的数据传输能力。

2） LTE-R 在 20M 可变带宽内, 支持上行峰值速率 50Mbit/s，下行峰值速率 100Mbit/s，对应的频谱 效率上行 $2.5 \mathrm{bits} / \mathrm{s} / \mathrm{Hz}$ ，下行 $5 \mathrm{bit} / \mathrm{s} / \mathrm{Hz}$ 。

3） LTE-R 采用全 IP 的网络结构, 各种业务均通过 IP 分组交换, 且通过了系统设计和严格 QoS 机制, 保证实时业务的服务质量。

4） LTE-R 降低了无线网络的时延，用户面最小时延可达到 $5 \mathrm{~ms}$ ，控制面时延小于 $100 \mathrm{~ms}$ 。

5）支持 $2 \mathrm{G} 、 3 \mathrm{GWIfi}$ 等多种无线接入技术，互通能力较强。

LTE-R 采用了 OFDM 技术、MIM0 天线技术及 64QAM 等调制技术，从而使得其具有更高的频谱利用率、 更高的数据传输速率及更高的安全性和更低的时延。

OFDM 技术（正交频分复用），是 LTE 在空中接口下行链路主要采用的技术，充分降低了终端成本与发 射终端峰均功率比。除此之外, 在空中接口上行链路中采用 SC-FDMA 技术, 主要是在原基础上对信号进行 充分扩展，以此避免 OFDM 系统带来的一些问题。

MIMO 技术（多输入多输出），该技术运行是通过发射信号或者接收信号来提升自身的传输效率，从而 达到应用效果。不同于 OFDM 技术的是, MIMO 通信技术能在不增加带宽的基础上加大频谱利用效率，最终 能使得系统传输效率得到提升。

\section{2LTE-R 的网络结构}

LTE-R 对传统的 3G 网络架构进行了优化，网络结构更加扁平化，其网络结构由核心网 EPC 及接入网 
E-UTRAN 组成。其网络结构如图 2 所示。

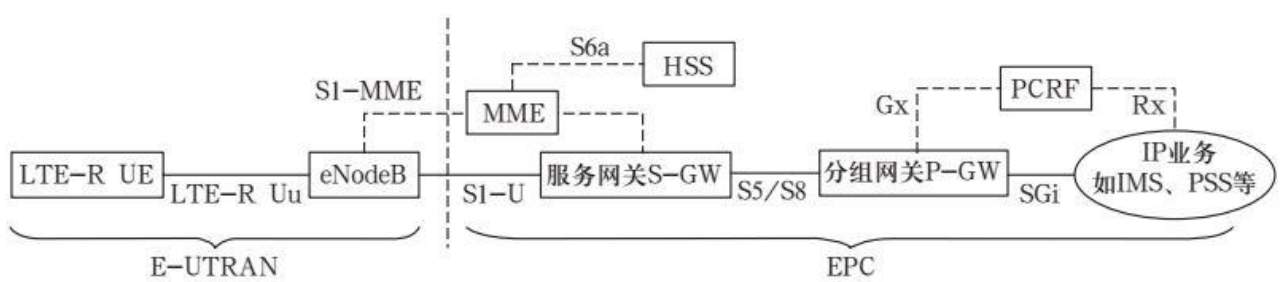

图 2 LTE-R 网络架构

基站 eNodeB 与终端用户设备（UE）共同组成了接入网 E-UTRAN。核心网 EPC 是由服务网关 S-GW、分 组网关 P-GW 所组成。LTE 的主要接口如下: 连接核心网与 eNodeB 的 SI 接口, 实现 eNodeB 之间的 X2 接口, 以及用户接入到系统的固定接口 LTE-Uu 接口。

eNodeB 主要功能为接收移动终端数据信息及部分无线资源的管理。同时 eNodeB 还负责将 IP 压缩加密, 终端用户设备 (UE) 附着移动管理实体 (MME) 的选择功能。此外, 还包括路由功能、上行传输层数据包 的分类标示、寻呼功能、广播功能等等。

MME 的主要功能是管理 NAS 信令以及其安全性。同时 MME 还负责将 3GPP 接入网络之间的核心网节点移 动性置零及漫游跟踪区列表管理。 $\mathrm{S}-\mathrm{GW}$ 主要功能为 eNOdeB 间切换本地和 $3 \mathrm{GPP}$ 间移动性针点，与 $2 \mathrm{G} 、 3 \mathrm{G}$ 网络互连进行针定, 下行分组缓冲、移动性管理及网络初始化等。 P-GW 可实现 IP 地址系统分配, 对上下 行数据进行分类，对现有数据包信息进行过滤。

\section{3LTE-R 与 GSM-R 的对比分析}

LTE-R 与 GSM-R 基础的技术参数比较如表 1 所示。

表 1 GSM-R 与 LTE-R 技术参数比较

\begin{tabular}{ccc}
\hline 技术性能 & LTE-R & GSM-R \\
\hline 工作频带 & $1.8 \mathrm{GHz} 、 2.3 \mathrm{GHz} 、 2.6 \mathrm{GHz}$ & $900 \mathrm{MHz}$ \\
带宽 & $1.25 \mathrm{MHz} 、 1.6 \mathrm{MHz} 、 2.5 \mathrm{MHz} 、$ & $200 \mathrm{KHz}$ \\
& $5 \mathrm{MHz} 、 15 \mathrm{MHz} 、 20 \mathrm{MHz}$ & \\
数据传输速率 & 上行 $50 \mathrm{Mbit} / \mathrm{s}$, 下行 $100 \mathrm{Mbit} / \mathrm{s}$ & $\mathrm{CSD}$ 速率 $9.6 \mathrm{kbit} / \mathrm{s}$ \\
& & $\mathrm{GPRS}$ 速率 $170 \mathrm{kbit} / \mathrm{s}$ \\
频谱效率 & 0.2 & 2 \\
最大时延 & $100 \mathrm{~ms}$ 以下 & $500 \mathrm{~ms}$ 左右 \\
多址方式 & OFDM & TDMA \\
调制方式 & QPSK、16QAM、64QAM & GMSK、8PSK \\
\hline
\end{tabular}

GSM-R 系统本身为窄带系统, 能承载对网络带宽要求不高的铁路业务, 如调度命令、车次号、列车速 度等信息, 而 LTE-R 系统支持广播单频网络, 因此可以使用 LTE 网络承担上述服务之外的服务, 例如移动 电视、视频监控等对于带宽要求较高的业务。

GSM-R 系统分为交换中心、基站控制器、基站及终端四个部分, 而 LTE-R 系统只分为三个部分, 没有 基站控制器, 因此使得网络结构更加简单, 降低了系统的复杂度, 减少了网络节点, 降低了建设和维护成 
本。

因为 LTE 系统与 GSM 系统相比, 减少了基站控制器部分, 原先的电路交换及分组交换结合网络简化 为全 IP 扁平化基础网络结构。 LTE 具有 E-UTRA 空中接口, LTE 网络在 E-UTRAN 环境下借助 QOS 技术可以 实现低于 $5 \mathrm{~ms}$ 的延迟。具体网络架构对比如图 3 所示。
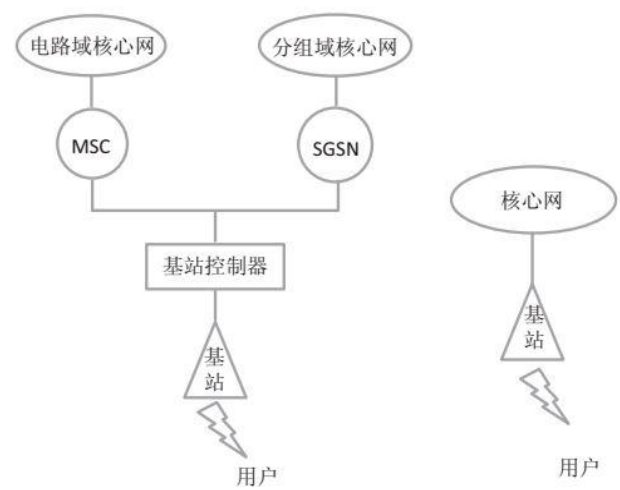

图 3 LTE-R 与 GSM-R 网络架构对比

GSM-R 系统立足于二代窄带技术, 自身数据承载能力有限, 仅能承载流量较小的专用数据。而 LTE-R 系统引入了 OFDM 和 MIMO 等技术, 增加了频谱效率和数据传输速率, 并支持了多种带宽分配, 支持全球主 流的频段和另外一些新增频段, 因此频谱分配更加灵活。

\section{LTE-R 技术用于高铁通信的展望}

LTE 作为准 $4 \mathrm{G}$ 的移动通信技术, 目前已经推广使用。LTE 技术的不断成熟, 也为了 LTE-R 系统在高铁 领域的业务应用提供了强有力的保证。

\section{1 提供现有 GSM-R 系统的业务}

LTE-R 系统作为 GSM-R 的长期演进项目, 不仅能够提供目前 GSM-R 系统所有现有的铁路业务应用, 如 调度信息、实时监控与控制信息、区间段工务信息、应急指挥信息等。LTE-R 所具有的低时延, 可以使其 传送信息的实时性更好, 从而保障了列车的运行安全, 调度信息更为及时, 遇到突发状况可以更快地做出 反应。

\section{2 实时视频图像传输业务}

LTE-R 作为宽带的无线通信系统, 使得其不仅可以实现调度时的语音通信, 还可以实时传输视频和图 像, 这样可以实时监控重点路段。通过车一地之间的视频传输, 可以实现实时监测列车运行状态, 车内重 点区域的监控, 也可以使得地面工作人员了解列车的运行状况。除此之外, 司机也可以收到沿线铁路设施 和车站、路口的实时状况, 这样司机就可以对沿途的运行状况作出判断, 并合适地调整运行状态。

另外, 一旦出现紧急情况, 地面调度中心可以通过 LTE 系统受到应急监控视频, 及时了解到现场情况, 从而做出最优判断。

\section{3 列车状态监测及预警}

列车运行安全监控系统是保障列车运行安全的重要方式, 在列车所有关键位置, 如车门、温度传感器、 制动系统、空调系统、电力系统、车速、温度等进行监控, 实时传送到地面调度中心, 为告诉运行的列车 提供安全运营及快速检修提供技术保障。

利用 LTE-R 网络, 可以实现全网列车实时监控, 通过对同一线路不同区间上各列车间距进行实时监控, 
一旦达到预警距离，对相关列车发出警报并通知控制中心。

\section{4 旅客移动信息业务}

随着移动通信技术的发展, 人们对于无线通信的需求日益增加。目前 GSM-R 系统还没有开通旅客信息 服务的业务, 列车上的通信均是由共用网络提供的。随着列车运行速度提高, 公用网络很难为旅客提供高 服务质量的随时随地的接入服务。

而利用 LTE-R 的高速率、低时延以及高移动性可为列车上旅客提供移动信息服务, 如视频、电影、在 线音乐等, 可以很好地提供互联网服务。

\section{4 结语}

综上所述, 相对于已经应用的 GSM-R 技术, LTE-R 技术有着众多优点, 如时延小、数据传输速率高、 工作频带宽等优点, 因此其有着非常广阔的应用前景。随着时代的进步, LTE-R 技术在铁路行业中的应用 是必然的, 它不仅可以保障铁路运行安全, 也可以满足铁路乘客的需求, 但是 LTE-R 技术形成系统并适用 于我国网络仍需一个较长的过程。

\section{参考文献}

[1]卜爱琴．铁路下一代移动通信技术 LTE-R 应用的探讨 [J]．信息通信，2014(2)：174-176.

[2]刘洪申. 浅析铁路新一代无线通信技术 LTE-R 的应用及发展 [J]。通讯世界，2015(11)：41-42.

[3]夏云琦. 铁路无线通信技术向 LTE-R 的演进 $[J]$. 中国铁路，2012 (8)：75-76.

［4]葛淑云，华尧．基于 LTE 制式的铁路宽带业务应用与关键技术研究 [J]．铁路通信信号工程技术，2013，(2)：14-16.

[5]Stefania Sesia, Lassm Toufik, Matthew Baker. LTE The UMTS Long Term Evolution From Theory to Practise(2nd Edition) [M]. UK: John Wiley\&Sons Ltd, 2011.

\section{References:}

[1] Bu Aiqin Discussion on LTE - R Application of Next Generation Mobile Communication Technology in Railway Indusry [J]. Information and Communication, 2014 (2): 174-176.

[2] Liu Hongshen Analysis on the Application and Development of Railway New Generation Wireless Communication Technology [J]. Communications World, 2015 (11): 41-42.

[3] Xia Yunqi Evolution of Railway Wireless Communication Technology to LTE - R [J]. China Railway, 2012 (8): 75-76.

[4] Ge Shuyun, Huayao Research on Railway Broadband Service Application and Key Technology Based on LTE System [J]. Railway Communication Signal Engineering, 2013, (2): 14-16. 